\title{
Left ventricle unloading during veno-arterial extracorporeal membrane oxygenation
}

\author{
Svitlana Strunina $\mathrm{MSc}^{1,2}$, Petr Ostadal MD PhD
}

S Strunina, P Ostadal. Left ventricle unloading during veno-arterial extracorporeal membrane oxygenation. Curr Res Cardiol 2016;3(1):5-8.

Extracorporeal membrane oxygenation in veno-arterial configuration (VA-ECMO) is a method widely used to support circulation during the most severe conditions of heart failure or refractory cardiac arrest. It frequently bridges this otherwise fatal status to recovery, therapeutic intervention or other circulatory support. However, the use of VA-ECMO in critical situations of severely impaired heart function is associated with specific limitations. One common complication is left ventricular overload and distension, primarily due to the increased afterload caused by extracorporeal blood flow. To date, various techniques has been proposed to decrease left ventricular distension and improve its function, including insertion of intra-aortic balloon pump or percutaneous left ventricular assist device, left atrial and left ventricular venting, or switch to surgically inserted left ventricular support with or without oxygenator. The present review summarizes current minimally invasive approaches to unload overloaded left ventricle during VA-ECMO support.

Key Words: Cardiogenic shock; Extracorporeal membrane oxygenation; Heart failure; Left ventricular overload; Left ventricular unloading

\section{Cxtracorporeal membrane oxygenation in veno-arterial configura-} Etion (VA-ECMO) provides right atrium-to-aortic circulatory support. The VA-ECMO circuit consists of a venous inflow cannula, an extracorporeal blood pump, an oxygenator, an arterial outflow cannula and connecting tubes. In minimally invasive (ie, peripheral) settings, the inflow cannula is usually inserted percutaneously from the femoral or jugular vein into the right atrium. The deoxygenated venous blood is drawn from the right atrium and is pumped by a continuous flow axial or centrifugal pump to the oxygenator where blood gases are exchanged. The oxygenated blood is then returned to the aorta via an arterial outflow cannula that is usually inserted into the femoral artery (Figure 1). The gas blender enables adjustment of the gas flow and oxygen fraction in the in the oxygenator. A heat exchanger can be connected to the oxygenator, thereby enabling temperature control in VA-ECMO-treated patients. Based on the cannula's size, the extracorporeal blood flow in VA-ECMO may reach up to $7 \mathrm{~L} / \mathrm{min}$ and, could therefore, partially support or even fully substitute for the cardiac pump and pulmonary gas exchange. Placement of VA-ECMO does not necessarily require fluoroscopic guidance and, in emergent situations, can be performed quickly at the patient bedside or in the field.

\section{CLINICAL INDICATIONS FOR VA-ECMO}

Currently, there are no randomized controlled trials focused on the clinical outcomes of VA-ECMO. However, several analyses of registries and case series have demonstrated promising results for VA-ECMO therapy. The major indication for VA-ECMO placement is severe or rapidly progressing cardiogenic shock. Combes et al (1) reported experience from a group of 81 consecutive patients in whom VA-ECMO was inserted due to refractory cardiogenic shock. Thirty-four $(42 \%)$ patients suvived despite a predicted mortality rate of nearly $100 \%$ before VA-ECMO placement. Sheu et al (2) described a retrospective comparison of 219 VA-ECMO-treated individuals with cardiogenic shock due to myocardial infarction with an historical control group of 115 patients. They found a significantly higher 30 -day survival rate in the VA-ECMO-treated group (60\%) than in the control group (35\%). Moreover, Beurtheret et al (3) demonstrated that VA-ECMO can be inserted in patients with refractory cardiogenic shock in remote institutions and safely transferred on ECMO to the tertiary-care centre: thirty-two (37\%) of 87 eligible patients with VA-ECMO insertion in remote hospitals survived to hospital

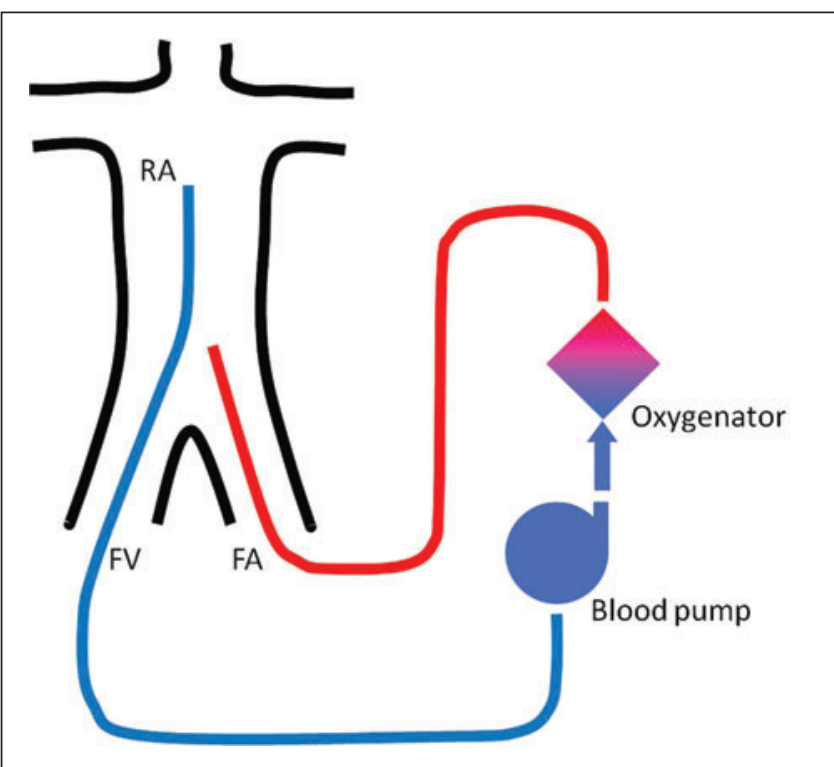

Figure 1) Veno-arterial extracorporeal membrane oxygenation in femorofemoral configuration. FA Femoral artery; FV Femoral vein; RA Right atrium

discharge. Survival following the insertion of VA-ECMO for cardiogenic shock remains poor; however, it must be considered that in many of these cases, the predicted mortality rate before ECMO implantation exceeded $90 \%$.

Refractory cardiac arrest represents another situation in which VA-ECMO is increasingly used. Insertion of ECMO during continuous cardiopulmonary resuscitation is also known as extracorporeal cardiopulmonary resuscitation (ECPR). Approximately one-half of patients experiencing resuscitated cardiac arrest die without return of spontaneous circulation $(4,5)$. ECPR may represent the last chance for survival in these patients. Currently, there are controversial reports regarding VA-ECMO insertion for refractory cardiac arrest. Megarbane et al (6) reported long-term survival in three of 17 patients with refractory cardiac arrest and ECPR. Le Guen (7) reported that only two patients survived with good neurological outcome from 51 treated

${ }^{1}$ Cardiovascular Center, Na Homolce Hospital; ${ }^{2}$ Faculty of Biomedical Engineering, Czech Technical University in Prague, Prague, Czech Republic Correspondence: Dr Petr Ostadal, Cardiovascular Center, Na Homolce Hospital, 15030 Prague, Czech Republic. Telephone 420257272208, fax420257272974, e-mail ostadal.petr@gmail.com 
with ECPR for refractory cardiac arrest. Conversely, Chen et al (8) observed an acceptable survival rate of $32 \%$ in 57 patients treated with ECPR. It appears that the outcomes of ECPR are worsening with the increasing time from collapse to ECMO initiation (9).

Several case series reported the use of VA-ECMO as support for high-risk percutaneous coronary and valvular intervention (10), arrhythmic storm, or electro-anatomical mapping and catheter ablation of nontolerated ventricular tachycardia (11). VA-ECMO has also been successfully used in sepsis-associated cardiomyopathy $(12,13)$, pulmonary hypertension (14) and pulmonary embolism $(15,16)$.

\section{LEFT VENTRICULAR OVERLOAD DURING VA-ECMO THERAPY}

VA-ECMO currently represents the most efficient minimally invasive circulatory support system. It provides sufficient support to enable adequate tissue perfusion even in cardiac arrest, and the hemodynamic efficacy is superior to other available percutaneous circulatory support systems such as Impella 2.5 (Abiomed Inc, USA) or TandemHeart ${ }^{\mathrm{TM}}$ (CardiacAssist, USA) (17). However, marked increase in systemic blood pressure caused by VA-ECMO may also impact left ventricular (LV) function. Increased LV afterload, together with severe systolic dysfunction, may result in LV overload with subsequent increase in left atrial pressure and severe pulmonary edema. It has been shown both in animal (18) and human (19) studies that increasing extracorporeal blood flow with VA-ECMO impairs several parameters of LV performance. VA-ECMO, therefore, represents a circulatory rather than LV assist device, and LV overload during VA-ECMO therapy represents a critical condition that frequently requires urgent intervention to unload the left ventricle. The present article summarizes current minimally invasive approaches to unload an overloaded left ventricle.

In the presence of severe LV dysfunction, the left ventricle is unable to eject a sufficient volume of blood against the increased afterload caused by the ECMO flow, resulting in impairment of various parameters of LV performance (18-20) and, in extreme situations, the aortic valve can remained closed even during systole. This results in LV overload with distension, increased wall stress and increased myocardial oxygen consumption (18-23). If cardiogenic shock has occurred as a result of decompensation in chronic heart failure, the left ventricle is likely to be compliant and the mitral valve is frequently incompetent as a result of chronic annular dilation and mitral valve leaflet tethering. The resultant mitral reflux would decompress the left ventricle to some extent, but may result in elevation of left atrial pressure and pulmonary edema $(20,21,23,24)$. In contrast, in acute cardiogenic shock, such as following acute myocarditis or myocardial infarction, the left ventricle is likely to be noncompliant and the mitral valve likely to be competent. LV distension in this setting will result in a significant rise in intraventricular pressure and wall tension, which could be detrimental to damaged myocardium. In addition, the rise in LV pressure could reduce coronary blood flow, causing myocardial ischemia, particularly in the subendocardial area $(20,25)$. The development of LV overload and distension could also be potentiated by the presence of aortic regurgitation (26).

\section{LEFT VENTRICLE UNLOADING}

To date, several approaches have been proposed to unload an overloaded left ventricle and decrease the elevated left atrial pressure on VA-ECMO therapy.

\section{Atrial septostomy}

Effective decompression of the left ventricle in the setting of VA-ECMO can be achieved by left-to-right shunt at the presence of atrial communication (atrial septal defect or patent formanen ovale); atrial shunt can be, however, created also artificially with percutaneous blade or balloon septostomy $(22,24)$. Seib et al (24) reported series of 10 patients with severe LV dysfunction (seven myocarditis, three dilated cardiomyopathy) who required circulatory support with ECMO and who underwent left heart decompression with blade and balloon atrial septostomy (BBAS). BBAS was performed while on $\mathrm{ECMO}$ in seven patients and pre-ECMO in three. A femoral venous approach was used in all patients. Trans-septal puncture was required in nine patients while one patient had a patent foramen ovale. The procedure was successful in all patients and led to LV decompression and pulmonary decongestion. Left atrial mean pressure fell from a mean of $30.5 \mathrm{mmHg}$ to $16 \mathrm{mmHg}$. Left atrial to right atrial pressure gradient fell from a mean of $20 \mathrm{mmHg}$ pre-BBAS to $3 \mathrm{mmHg}$ post-BBAS. Artificial atrial septal defect size ranged from $2.5 \mathrm{~mm}$ to $8 \mathrm{~mm}(24)$.

\section{Left atrial venting}

With guidance by bedside transoesophageal echocardiography, a percutaneous atrial trans-septal cannula can be placed and connected to the inflow part of the ECMO circuit, thus, decompressing the left ventricle $(27,28)$. Swartz et al $(27)$ reported a case involving a 13-year-old girl who presented with cardiogenic shock. VA-ECMO was initiated, but after six days, severe LV distension resulted in decreased VA-ECMO flows. With guidance by bedside transesophageal echocardiography, a percutaneous atrial trans-septal cannula was placed and connected to the venous circuit, thus decompressing the left ventricle. The patient improved, was weaned from VA-ECMO five days later and was discharged from the hospital (27). Aiyagari et al (28) described a series of seven patients with cardiac failure on VA-ECMO with left atrial hypertension. All patients underwent left atrial decompression with transseptal puncture and placement of a drain ( 8 Fr to $15 \mathrm{Fr}$ ). The median time from ECMO cannulation to left atrial decompression was $11 \mathrm{~h}$. Average initial left atrial pressure was $31 \mathrm{mmHg}$. Successful drain placement was achieved in all patients with no major periprocedural complications. Echocardiographic improvement in left atrial dilation was achieved in five $(71 \%)$ patients. Inability to decompress the left atrium was fatal in two patients. Four (57\%) patients were decannulated and three (43\%) survived to hospital discharge (28).

\section{LV venting}

The left ventricle can be vented directly by placing a transaortic vent through the axillary artery or by echocardiography-guided insertion of a pigtail catheter into the left ventricle through the aortic valve and connected to the inflow part of the ECMO circuit $(29,30)$. Fumagalli et al (29) reported a case in which extracorporeal life support for cardiogenic shock was complemented with ventricular decompression achieved with a catheter placed percutaneously through the aortic valve into the left ventricle. The blood drained from the left ventricle was pumped into the femoral artery. The normalization of left heart filling pressures led to the resolution of pulmonary edema, and the patient underwent a successful heart transplantation after seven days of mechanical cardiocirculatory support (29). Barbone et al (30) proposed LV unloading with a 7 Fr pigtail catheter inserted into the left ventricle via the femoral artery contralateral to the arterial outflow cannula. Using this approach, they were able to support three different patients, resolving LV distension and preventing lung congestion without major complications.

\section{Pulmonary artery drainage}

An alternative approach to LV decompression is the percutaneous insertion of a venous cannula into the pulmonary artery and connection of this cannula to the inflow part of the ECMO circuit $(23,31)$. Avali et al (31) reported a case involving a 43-year-old woman treated with ECMO for refractory cardiogenic shock after left pneumonia and severe sepsis. A 15 Fr venous cannula was placed percutaneously to the pulmonary artery and connected to the ECMO circuit to decompress the left heart, and to facilitate LV function. After myocardial recovery, the patient was weaned and ECMO was removed on day 16 (31). Fouilloux et al (23) described unloading of the left ventricle with a cannula inserted into the pulmonary trunk through the inferior vena cava with a femoral approach in two-year-old girl with a restrictive cardiomyopathy. A few hours later, the chest $\mathrm{x}$-ray improved and five 
days later, recovery of the left ventricular function allowed removal of the pulmonary artery cannula (23).

\section{INTRA-AORTIC BALLOON PUMP}

Intra-aortic balloon pump (IABP) counter-pulsation is a device that inflates and deflates a $30 \mathrm{~cm}$ to $50 \mathrm{~cm}$ balloon in the descending aorta. The balloon inflations and deflations are synchronized with cardiac cycle and, therefore, deflation just before systolic ejection may decrease afterload and improve LV ejection. Moreover, increased diastolic pressure on IABP could also improve coronary blood flow. Despite the controversial data from the Intra-Aortic Balloon Pump in cardiogenic SHOCK (IABP-SHOCK) II trial (32), IABP currently remains one of the most commonly used mechanical circulatory support devices in the treatment of acute heart failure. When administered in a timely manner, it can play a critical role in the rescue of patients with acute myocardial damage. It has been shown in animal models that insertion of IABP during VA-ECMO support may improve several parameters of LV performance (33). Currently, several centres use IABP to reduce LV afterload during VA-ECMO therapy. In a group of 219 patients treated with VA-ECMO after cardiac surgery, Doll et al (34) found that use of IABP during ECMO support was associated with a significantly higher survival rate. Ma et al (35) reported 54 adult patients with acute heart failure who received combined ECMO and IABP support, all of whom showed improvements in terms of overall circulation. Thirty-four of the patients were successfully weaned from mechanical circulatory support, and 21 (39\%) survived to hospital discharge (35). The study by Petroni et al (36) showed that adding an IABP to peripheral VA-ECMO was associated with improved LV function. Discontinuation of intra-aortic balloon pumping was associated with higher pulmonary artery wedge pressure $(19 \pm 10$ versus $15 \pm 8 \mathrm{mmHg} ; \mathrm{P}=0.01)$, increased LV end-systolic ( $51 \pm 13$ versus $50 \pm 14 \mathrm{mmHg} ; \mathrm{P}=0.05)$ and end-diastolic ( $55 \pm 13$ versus $52 \pm 14 \mathrm{mmHg} ; \mathrm{P}=0.003)$ diameters, and decreased pulse pressure $(15 \pm 13$ versus $29 \pm 22 \mathrm{mmHg} ; \mathrm{P}=0.02)$ (36). In contrast, Park et al (37) did not find any mortality or morbidity benefit with IABP in the group

\section{REFERENCES}

1. Combes A, Leprince P, Luyt CE, et al. Outcomes and long-term quality-of-life of patients supported by extracorporeal membrane oxygenation for refractory cardiogenic shock. Crit Care Med 2008;36:1404-11.

2. Sheu JJ, Tsai TH, Lee FY, et al. Early extracorporeal membrane oxygenator-assisted primary percutaneous coronary intervention improved 30-day clinical outcomes in patients with ST-segment elevation myocardial infarction complicated with profound cardiogenic shock. Crit Care Med 2010;38:1810-7.

3. Beurtheret S, Mordant P, Paoletti X, et al. Emergency circulatory support in refractory cardiogenic shock patients in remote institutions: A pilot study (The Cardiac-RESCUE Program). Eur Heart J 2013;34:112-20.

4. Nolan JP, Neumar RW, Adrie C, et al. Post-cardiac arrest syndrome: Epidemiology, pathophysiology, treatment, and prognostication. A Scientific Statement from the International Liaison Committee on Resuscitation; the American Heart Association Emergency Cardiovascular Care Committee; the Council on Cardiovascular Surgery and Anesthesia; the Council on Cardiopulmonary, Perioperative, and Critical Care; the Council on Clinical Cardiology; the Council on Stroke. Resuscitation 2008;79:350-79.

5. Neumar RW, Nolan JP, Adrie C, et al. Post-cardiac arrest syndrome: Epidemiology, pathophysiology, treatment, and prognostication. A consensus statement from the International Liaison Committee on Resuscitation (American Heart Association, Australian and New Zealand Council on Resuscitation, European Resuscitation Council, Heart and Stroke Foundation of Canada, InterAmerican Heart Foundation, Resuscitation Council of Asia, and the Resuscitation Council of Southern Africa); the American Heart Association Emergency Cardiovascular Care Committee; the Council on Cardiovascular Surgery and Anesthesia; the Council on Cardiopulmonary, Perioperative, and Critical Care; the Council on Clinical Cardiology; and the Stroke Council. Circulation. 2008;118:2452-83 of 96 VA-ECMO-treated patients with cardiogenic shock due to acute myocardial infarction.

\section{PERCUTANEOUS LV SUPPORT DEVICES}

Impella LP 2.5 (Abiomed Inc, USA) is a catheter-based transaortic axial flow pump that can be introduced through a percutaneous femoral approach. The device is placed across the aortic valve and pumps up to $2.5 \mathrm{~L} / \mathrm{min}$ of blood from the left ventricle to the ascending aorta. Koeckert et al (25) reported the use of Impella LP 2.5 for left ventricle decompression in a 70-year-old man with decompensated heart failure who was placed on VA-ECMO for cardiogenic shock with severe pulmonary edema and respiratory failure. Both devices were successfully weaned on day 5 after myocardial recovery (25). Narain et al (38) described a case involving 31-year-old man with fulminant myocarditis treated with the Impella device and VA-ECMO. On full mechanical circulatory support, the hemodynamic status improved and both systems were explanted after $48 \mathrm{~h}$ (38).

\section{CONCLUSIONS}

Despite the advances in critical care during the past years, the mortality rate associated with rapidly progressing or severe refractory cardiogenic shock remains high and mechanical circulatory support is often the last chance for survival. An increasing body of evidence supports the use of VA-ECMO in these critical conditions. However, especially in patients with most severe heart dysfunction, initiation of VA-ECMO may be associated with LV overload with all of its consequences including severe pulmonary edema and respiratory failure. Current options for minimally invasive LV unloading during VA-ECMO therapy are very limited; however, to date, several techniques have been described with promising results.

ACKNOWLEDGEMENTS: This work was supported by an Institutional grant MH CZ - DRO (Nemocnice Na Homolce - NNH, 00023884).

6. Megarbane B, Leprince P, Deye N, et al. Emergency feasibility in medical intensive care unit of extracorporeal life support for refractory cardiac arrest. Intensive Care Med 2007;33:758-64.

7. Le Guen M, Nicolas-Robin A, Carreira S, et al. Extracorporeal life support following out-of-hospital refractory cardiac arrest. Crit Care 2011;15:R29.

8. Chen YS, Chao A, Yu HY, et al. Analysis and results of prolonged resuscitation in cardiac arrest patients rescued by extracorporeal membrane oxygenation. J Am Coll Cardiol 2003;41:197-203.

9. Wang $\mathrm{CH}$, Chen YS, Ma MH. Extracorporeal life support. Curr Opin Crit Care 2013;19:202-7.

10. Arlt M, Philipp A, Voelkel S, et al. Early experiences with miniaturized extracorporeal life-support in the catheterization laboratory. Eur J Cardiothorac Surg 2012;42:858-63.

11. Rizkallah J, Shen S, Tischenko A, Zieroth S, Freed DH, Khadem A Successful ablation of idiopathic left ventricular tachycardia in an adult patient during extracorporeal membrane oxygenation treatment. Can J Cardiol 2013;29:1741, e17-9.

12. Brechot N, Luyt CE, Schmidt M, et al. Venoarterial extracorporeal membrane oxygenation support for refractory cardiovascular dysfunction during severe bacterial septic shock. Crit Care Med 2013;41:1616-26.

13. Huang CT, Tsai YJ, Tsai PR, Ko WJ. Extracorporeal membrane oxygenation resuscitation in adult patients with refractory septic shock. J Thorac Cardiovasc Surg 2013;146:1041-6.

14. Abrams DC, Brodie D, Rosenzweig EB, Burkart KM, Agerstrand CL, Bacchetta MD. Upper-body extracorporeal membrane oxygenation as a strategy in decompensated pulmonary arterial hypertension. Pulm Circ 2013;3:432-5.

15. Maggio P, Hemmila M, Haft J, Bartlett R. Extracorporeal life support for massive pulmonary embolism. J Trauma 2007;62:570-6.

16. Munakata R, Yamamoto T, Hosokawa Y, et al. Massive pulmonary embolism requiring extracorporeal life support treated with catheter-based interventions. Int Heart J 2012;53:370-4 
17. Ostadal P, Mlcek M, Holy F, et al. Direct comparison of percutaneous circulatory support systems in specific hemodynamic conditions in a porcine model. Circ Arrhythm Electrophysiol 2012;5:1202-6.

18. Ostadal P, Mlcek M, Kruger A, et al. Increasing venoarterial extracorporeal membrane oxygenation flow negatively affects left ventricular performance in a porcine model of cardiogenic shock. J Transl Med 2015;13:266.

19. Aissaoui N, Guerot E, Combes A, et al. Two-dimensional strain rate and Doppler tissue myocardial velocities: Analysis by echocardiography of hemodynamic and functional changes of the failed left ventricle during different degrees of extracorporeal life support. J Am Soc Echocardiogr 2012;25:632-40.

20. Soleimani B, Pae WE. Management of left ventricular distension during peripheral extracorporeal membrane oxygenation for cardiogenic shock. Perfusion 2012;27:326-31.

21. Massetti M, Gaudino M, Crea F. How to transform peripheral extracorporeal membrane oxygenation in the simplest mid-term paracorporeal ventricular assist device. Int J Cardiol 2013;166:551-3.

22. Kotani Y, Chetan D, Rodrigues W, et al. Left atrial decompression during venoarterial extracorporeal membrane oxygenation for left ventricular failure in children: Current strategy and clinical outcomes. Artif Organs 2013;37:29-36.

23. Fouilloux V, Lebrun L, Mace L, Kreitmann B. Extracorporeal membranous oxygenation and left atrial decompression: A fast and minimally invasive approach. Ann Thorac Surg 2011;91:1996-7.

24. Seib PM, Faulkner SC, Erickson CC, et al. Blade and balloon atrial septostomy for left heart decompression in patients with severe ventricular dysfunction on extracorporeal membrane oxygenation. Catheter Cardiovasc Interv 1999;46:179-86.

25. Koeckert MS, Jorde UP, Naka Y, Moses JW, Takayama H. Impella LP 2.5 for left ventricular unloading during venoarterial extracorporeal membrane oxygenation support. J Card Surg 2011;26:666-8.

26. Sidebotham D, Allen S, McGeorge A, Beca J. Catastrophic left heart distension following initiation of venoarterial extracorporeal membrane oxygenation in a patient with mild aortic regurgitation. Anaesth Intensive Care 2012;40:568-9.

27. Swartz MF, Smith F, Byrum CJ, Alfieris GM. Transseptal catheter decompression of the left ventricle during extracorporeal membrane oxygenation. Pediatr Cardiol 2012;33:185-7.
28. Aiyagari RM, Rocchini AP, Remenapp RT, Graziano JN. Decompression of the left atrium during extracorporeal membrane oxygenation using a transseptal cannula incorporated into the circuit. Crit Care Med 2006;34:2603-6.

29. Fumagalli R, Bombino M, Borelli M, et al. Percutaneous bridge to heart transplantation by venoarterial ECMO and transaortic left ventricular venting. Int J Artif Organs 2004;27:410-3.

30. Barbone A, Malvindi PG, Ferrara P, Tarelli G. Left ventricle unloading by percutaneous pigtail during extracorporeal membrane oxygenation. Interact Cardiovasc Thorac Surg 2011;13:293-5.

31. Avalli L, Maggioni E, Sangalli F, Favini G, Formica F, Fumagalli R. Percutaneous left-heart decompression during extracorporeal membrane oxygenation: An alternative to surgical and transeptal venting in adult patients. ASAIO J 2011;57:38-40.

32. Thiele H, Zeymer U, Neumann FJ, et al. Intraaortic balloon support for myocardial infarction with cardiogenic shock. N Engl J Med 2012;367:1287-96

33. Sauren LD, Reesink KD, Selder JL, Beghi C, van der Veen FH, Maessen JG. The acute effect of intra-aortic balloon counterpulsation during extracorporeal life support: An experimental study. Artif Organs 2007;31:31-8.

34. Doll N, Kiaii B, Borger M, et al. Five-year results of 219 consecutive patients treated with extracorporeal membrane oxygenation for refractory postoperative cardiogenic shock. Ann Thorac Surg 2004;77:151-7.

35. Ma P, Zhang Z, Song T, et al. Combining ECMO with IABP for the treatment of critically Ill adult heart failure patients. Heart Lung Circ 2014;23:363-8.

36. Petroni T, Harrois A, Amour J, et al. Intra-aortic balloon pump effects on macrocirculation and microcirculation in cardiogenic shock patients supported by venoarterial extracorporeal membrane oxygenation. Crit Care Med 2014:42:2075-82.

37. Park TK, Yang JH, Choi SH, et al. Clinical impact of intra-aortic balloon pump during extracorporeal life support in patients with acute myocardial infarction complicated by cardiogenic shock. BMC Anesthesiol 2014;14:27.

38. Narain S, Paparcuri G, Fuhrman TM, Silverman RB, Peruzzi WT. Novel combination of impella and extra corporeal membrane oxygenation as a bridge to full recovery in fulminant myocarditis. Case Rep Crit Care 2012;2012:459296. 